\title{
An Analytical Approach for Deformation Shapes of a Cylindrical Shell with Internal Medium Subjected to Lateral Contact Explosive Loading
}

\author{
Xiangyu Li, Zhenduo Li, and Minzu Liang \\ College of Science, National University of Defense Technology, Changsha 410073, China \\ Correspondence should be addressed to Xiangyu Li; xiangyulee@nudt.edu.cn
}

Received 27 February 2015; Revised 18 June 2015; Accepted 24 June 2015

Academic Editor: Yuri Petryna

Copyright (c) 2015 Xiangyu Li et al. This is an open access article distributed under the Creative Commons Attribution License, which permits unrestricted use, distribution, and reproduction in any medium, provided the original work is properly cited.

An experimental investigation on deformation shape of a cylindrical shell with internal medium subjected to lateral contact explosion was carried out briefly. Deformation shapes at different covered width of lateral explosive were recovered experimentally. Based on the experimental results, a corresponding analytical approach has been undertaken with rigid plastic hinge theory. In the analytical model, the cylindrical shell is divided into end-to-end rigid square bars. Deformation process of the cylindrical shell is described by using the translations and rotations of all rigid square bars. Expressions of the spring force, buckling moment, and deflection angle between adjacent rigid square bars are conducted theoretically. Given the structure parameters of the cylinder and the type of the lateral explosive charge, deformation processes and shapes are reported and discussed using the analytical approach. A good agreement has been obtained between calculated and experimental results, and thus the analytical approach can be considered as a valuable tool in understanding the deformation mechanism and predicting the deformation shapes of the cylindrical shell with internal medium subjected to lateral contact explosion. Finally, parametric studies are carried out to analyze the effects of deformation shape, including the covered width of the lateral explosive, explosive charge material, and distribution of initial velocity.

\section{Introduction}

Cylindrical shells are used in a wide variety of engineering applications, from the containment pressure vessels of nuclear reactors to the bracing elements of aerospace structures. Such structures may be subjected to a wide variety of short duration transient loads throughout the course of their working life, such as air blasts, underwater explosions, and high velocity impact. Accurate prediction of the dynamic plastic deformation and rupture of the cylindrical shell subjected to high intensity transient loading is of great importance in many industrial applications.

Early researches on the dynamic buckling and failure of cylinders were restricted to axisymmetric external radial pulse loading [1] and axial impact loading. However, the corresponding analysis, though presented in an elegant analytical form, is of limited applicability because axisymmetric dynamic loading seldom occurs in practice. In real world situations loading is usually applied to one side of the cylinder and is characterized by various degrees of locality. It may consist of a projectile, missile or mass impact, standoff explosion described by a pressure pulse, or contact explosion often approximated as an ideal impulsive loading.

Depending on the load intensity and the special distribution of contact pressures, various forms of damage may result ranging from large amplitude lateral deflections to punchthrough penetration, fracture initiation at the base plate, progression of tearing fracture, and finally massive structural damage. Yakupov $[2,3]$ studied the dynamic response of the cylindrical shell subjected to a planar plastic shock wave with rigid plastic hinge theory and presented the residual deformation of the cylindrical shell as a function of a planar wave pressure. Gefken et al. [4] extended the earlier analysis by Lindberg and Florence to one-side inward radial pressure that varied as the cosine of the angular position around the shell and was uniform along length, to identify the structural 
response modes of thin cylindrical shell, with and without internal pressure, subjected to external radial impulsive loads. For unpressurized shells the response modes consisted of dynamic pulse buckling followed by large inward deflections of the loaded surface. In shell with high internal pressure, these response modes were followed by an outward motion driven by the internal pressure. Fatt and Wierzbicki [5] and Wierzbicki and Fatt [6] investigated the large amplitude transient response of plastic cylindrical shells using a stringon-foundation model, and the model incorporated two main load-resistance mechanisms in the shell: stretching in the longitudinal direction and bending in the circumferential direction. Jiang and Olson [7] presented a numerical model for large deflection, elastic-plastic analysis of the cylindrical shell structures under air blast loading condition based on a transversely curved finite strip formulation. Li and Jones [8] studied the dynamic response of a "short" cylindrical shell, which is made from a rigid, perfectly plastic material, and the plastic behavior of the material is controlled by the transverse shear force as well as the circumferential membrane and the longitudinal bending moment.

In recent years, increasing attention of both engineering communities and government agencies has turned to the dynamic response of the cylindrical shell subjected to underwater explosion. Pédron and Combescure [9] presented a modal method of analysis to determine the response of an infinitely long stiffened cylindrical shell of revolution lateral pressure produced by an underwater explosion and propagating in an acoustic fluid. Rajendran and Lee [10] studied comparative damage of air-backed and water-backed plates subjected to noncontact underwater explosion. Hung et al. [11] investigated the linear and nonlinear dynamic responses of three cylindrical shell structures subjected to underwater small charge explosion under different standoff distances. The three cylindrical shell structures were unstiffened, internally stiffened, and externally stiffened, respectively. Li and Rong [12] studied the dynamic response of the cylindrical shell structures subjected to underwater explosion by using experimental and numerical methods. Li et al. [13] investigated two kinds of the cylindrical shell models with the same geometry characteristics: unfilled and main hull sand-filled. The main hull sand-filled cylindrical shell is more difficult to be damaged by the shock wave loading than the unfilled model. Hu et al. [14] studied the effects of elastic modulus, shell radius, and thickness on the transient response characteristics of the cylindrical shell. Yao et al. [15] presented a new shock factor based on energy acting on the structure to describe the loading of underwater explosion. Jama et al. [16] reported an experimental and analytical investigation of steel square hollow sections subjected to transverse blast load, and the energy dissipated in the local deformation is discussed. In recent decades, the research on the performance of the cylindrical shell is still very limited. A series of analytical models have been developed, to predict the dynamic response of a cylindrical shell subjected to a lateral shock loading, or localized loading, or underwater explosion. Because of complexities introduced by unsymmetric loading, large displacements, and rotations of the shell amplified by material nonlinearities, the problem does not lend itself easily to an analytical treatment. However, very few studies have been reported on cylindrical shell filled with medium subjected to contact explosion loading.

To investigate the behavior of the cylindrical shell with internal medium loaded by lateral contact explosion, several experiments have been conducted, and the experimental results are presented and discussed in detail in this paper. Based on the experiments, a corresponding analytical approach was conducted with rigid plastic hinge theory. Deformation processes of cylindrical shells are described using the translations and rotations of all rigid square bars. In this study, an infinitely long cylindrical shell filled with medium subject to lateral contact explosion is performed. Because the cylindrical shell has the unique deformation shape in the symmetric axis direction, we take a ring representing the cylinder. Due to solving the inertia moment of the ring, a unit height ring represents deformation of the infinite cylinder. Because the ring has the same value of thickness and height in the radial and axial direction, the cross section of the ring in the circumferential direction is square, so we call it "square bar."

Given the structural parameters of the ring and the type of the explosive charge, deformation processes and shapes are reported using the analytical approach. A good agreement has been obtained between calculated and experimental results. Finally, parametric studies are carried out to analyze the effects of deformation shapes, depending on the covered width of the lateral explosive, explosive charge material, and distribution of initial velocity.

\section{Experimental Procedure and Results}

2.1. Experimental Procedure. In order to investigate the dynamic response of the cylindrical shell with internal medium subjected to lateral contact explosion loading, some experiments were carried out with respect to the different covered widths of the lateral explosive charge. The photography and assembly schematics of experimental setups are shown in Figures 1 and 2, respectively.

The experimental setup consists of a cylindrical shell with internal medium (sand), a lateral explosive charge, a connecting rod, and two endplates. The thickness $t$, the outer radius $r_{1}$, and the axial length $H$ of the cylindrical shell, made from 1020 Steel, are $2 \mathrm{~mm}, 100 \mathrm{~mm}$, and $220 \mathrm{~mm}$, respectively. The radius of the cylindrical shell is more than 10 times the thickness. The internal medium with a center hole reserved is local sand, having a density of $1.75 \mathrm{~g} / \mathrm{cm}^{3}$. Two endplates, made from LY12 Aluminium, having a thickness of $10 \mathrm{~mm}$, are fixed by the connecting rod so that fully closed condition will be simulated. Material properties of 1020 Steel, LY12 Aluminum, and sand are listed in Table 1.

The lateral charge is an emulsion explosive (DL103-80), which is made from 75\% PETN, 20\% emulsion, and 5\% $\mathrm{Pb}_{3} \mathrm{O}_{4}$, and the density is $0.95 \mathrm{~g} / \mathrm{cm}^{3}$. The emulsion explosive DL103-80 is a sort of mild and flexible material that can be easily shaped. The inner radius, the thickness, and the covered width of the lateral explosive charge are $100 \mathrm{~mm}, 5 \mathrm{~mm}$, and $\varphi$, respectively. The explosion of each test is initiated by an electric detonator on the top of the lateral explosive charge. 
TABLE 1: Material properties of 1020 Steel, LY12 Aluminium, and sand.

\begin{tabular}{lcccc}
\hline Material & Density $\left(\mathrm{g} / \mathrm{cm}^{3}\right)$ & Yield stress $(\mathrm{MPa})$ & Young's modulus $(\mathrm{GPa})$ & Poisson's ratio \\
\hline 1020 Steel & 7.85 & 275 & 210 & 0.29 \\
LY12 Aluminium & 2.78 & 230 & 70 & 0.29 \\
Sand & 1.75 & 4.23 & 0.01 & 0.26 \\
\hline
\end{tabular}

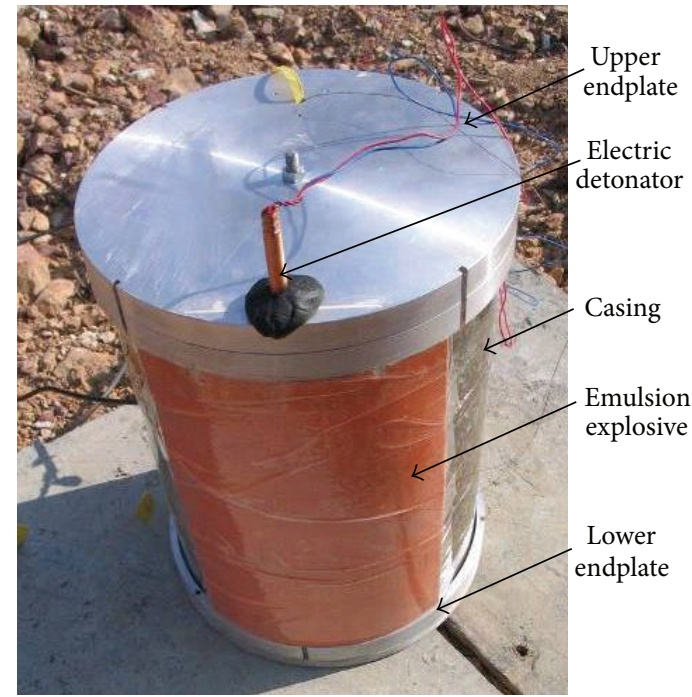

FIgURE 1: Photography of the experimental setups.

2.2. Experimental Results. Deformation shapes of the cylindrical shell with internal medium at three different covered widths of the lateral explosive charge were recovered. Deformation shapes after tests are shown in Figure 3. From the deformation shapes recovered, the angle of the lateral charge has a significant effect on the deformation shape, and the deformation shapes are concave, linear, and convex when the covered widths of lateral charges are $45^{\circ}, 90^{\circ}$, and $135^{\circ}$, respectively.

\section{Analytical Model}

3.1. Basic Assumptions. Deformation processes of the cylindrical shell with internal medium subjected to lateral contact explosion are a high nonlinear problem. Due to complexities introduced by unsymmetric loading, large displacements, and rotations of the cylinder amplified by material nonlinearities, the problem does not lend itself easily to an analytical treatment. However, by introducing a suitable set of assumptions, a simple and realistic model can be established to describe the deformation processes of the cylindrical shell with internal medium.

Basic assumptions are as follows: (1) the cylindrical shell is infinitely long, and the axial thickness selected is equal to the thickness of cylinder; (2) the cylinder is divided into endto-end rigid square bars along the circumferential direction; (3) the square bars close to the lateral explosive charges have an instantaneous velocity pointing to the centre of the cylinder.
During deformation processes of the cylindrical shell, some parameters of square bars may vary at different moments, such as translational displacements, translational velocities, rotational displacements, rotational angles, and area surrounded by square bars, which affect the value of the spring force, the bending moment, and the deflection angle intensively.

3.2. Analytical Approach. Based on the above-mentioned assumptions, the analytical model is established, shown in Figure 4, where the origins for $x$ and $y$ are given, and the range $x$ is from 0 to $2 r_{i}$, and the range $y$ is from $-r_{i}$ to $r_{i}$. The cylindrical shell is divided into end-to-end rigid square bars along the circumferential direction. The arc length $l$ of each square bar is $2 \pi r_{1} / N$ ( $N$ is the total number of square bars). The relationships between two adjacent square bars are established by using a spring force, a bending moment, and a deflection angle.

The spring force between adjacent square bars assumed as the perfect elastic-plastic is described by a changeable spring force (Figure 5). The spring force between adjacent square bars is expressed by the equation

$$
F= \begin{cases}E \cdot s \cdot t^{2} & s \leq s_{0} \\ \sigma_{y} \cdot t^{2} & s>s_{0},\end{cases}
$$

where $E, \sigma_{y}$, and $s_{0}$ are Young's modulus, the yield stress, and the elastic limit displacement of bar, respectively. $F$ is the spring force between adjacent square bars, and $s$ is the relative displacement between the end of the current bar and the head of the next bar.

The relative displacements between the end of the current bar and the head of the next bar are obtained by using the end displacement of the current square bar subtracting the head displacement of the next square bar. The relative displacements of adjacent square bars are described by the equation

$$
\begin{aligned}
s_{x}(i)= & {\left[d t \cdot v_{x}(i)-\frac{l \cdot \theta(i)}{2} \sin \alpha(i)\right] } \\
& -\left[d t \cdot v_{x}(i+1)-\frac{l \cdot \theta(i+1)}{2} \sin \alpha(i+1)\right], \\
s_{y}(i)= & {\left[d t \cdot v_{y}(i)+\frac{l \cdot \theta(i)}{2} \cos \alpha(i)\right] } \\
& -\left[d t \cdot v_{y}(i+1)+\frac{l \cdot \theta(i+1)}{2} \cos \alpha(i+1)\right],
\end{aligned}
$$

where $s_{x}(i)$ and $s_{y}(i)$ are relative displacements between two adjacent square bars in the $x$-axis and $y$-axis directions, 


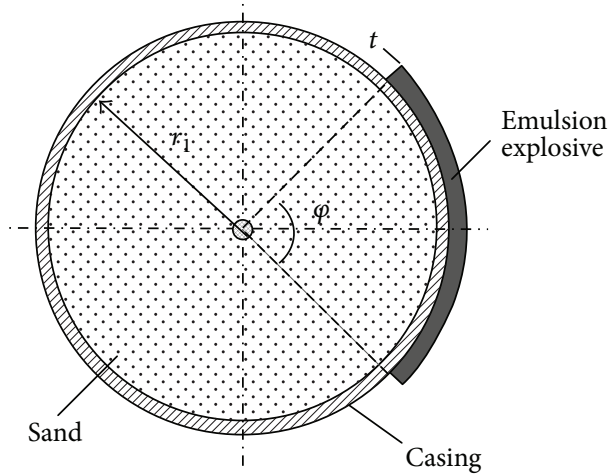

(a) Section drawing

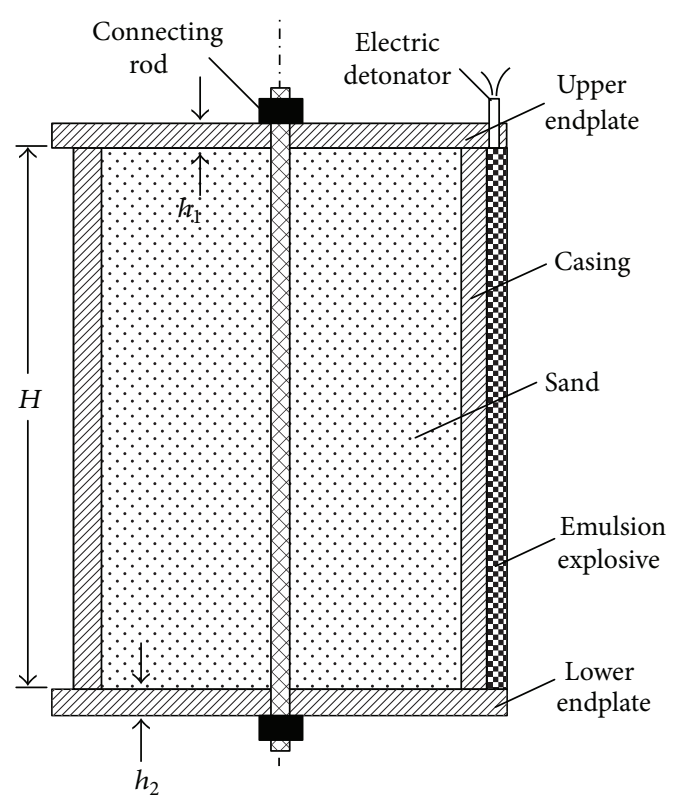

(b) Profile drawing

FIGURE 2: Assembly schematics of experimental setups: (a) section drawing and (b) profile drawing.

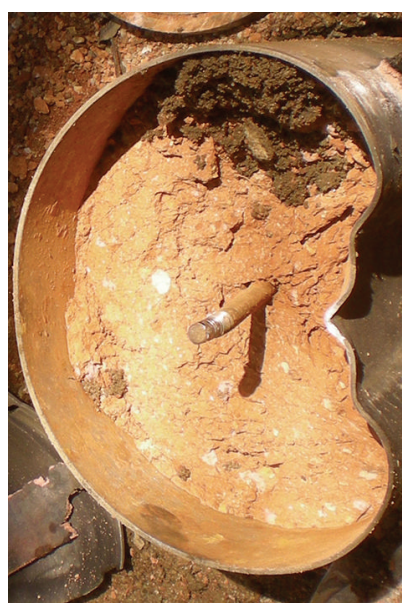

(a) $45^{\circ}$

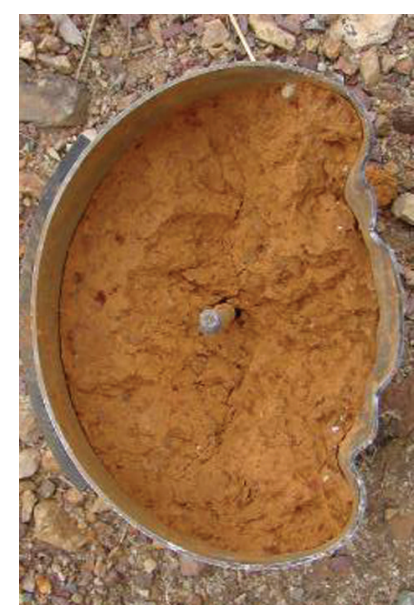

(b) $90^{\circ}$

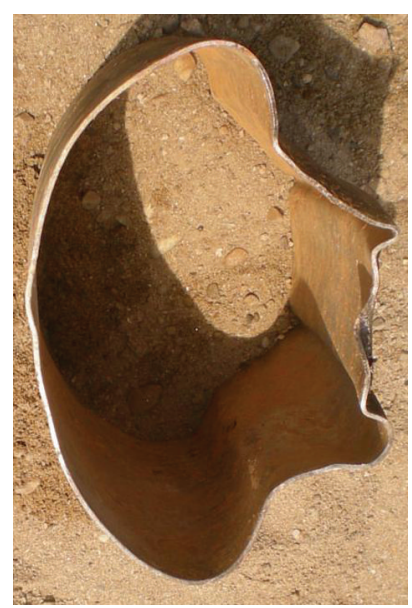

(c) $135^{\circ}$

FIGURE 3: Experimental result of deformation shapes after tests at three different covered widths of the lateral explosive charge.

respectively. $d t$ is a time step. $v_{x}(i)$ and $v_{y}(i)$ are translational velocities of the current square at the $x$-axis and $y$-axis directions, respectively. $v_{x}(i+1)$ and $v_{y}(i+1)$ are translational velocities of the next square at the $x$-axis and $y$-axis directions, respectively. $\theta(i)$ is the relative deflection angle between two adjacent square bars. The spring force between two adjacent square bars is calculated by the following equation when $s_{x}(i)$ and $s_{y}(i)$ are less than $s_{0}$ :

$$
\begin{aligned}
& F_{x}(i)=E \cdot t^{2} \cdot s_{x}(i), \\
& F_{y}(i)=E \cdot t^{2} \cdot s_{y}(i),
\end{aligned}
$$

where $F_{x}(i)$ and $F_{y}(i)$ are spring forces between two adjacent square bars in the $x$-axis and $y$-axis directions, respectively.

Bending moment and corresponding deflection angle between two adjacent square bars are shown in Figure 6 . Based on the rigid plastic hinge theory, the plastic hinges are achieved when the bending moment of the square bar reaches the plastic ultimate bending moment. During the deformation processes of the cylindrical shell, the relationships between the bending moment $M$ and the deflection angle $\theta$ are assumed to be linear when the bending moment is less than the plastic ultimate bending moment $M_{p}$. Meanwhile, the deflection $\theta$ is set to the plastic limit deflection angle $\theta_{p}$ when the bending moment is greater than or equal to the plastic ultimate bending moment. 


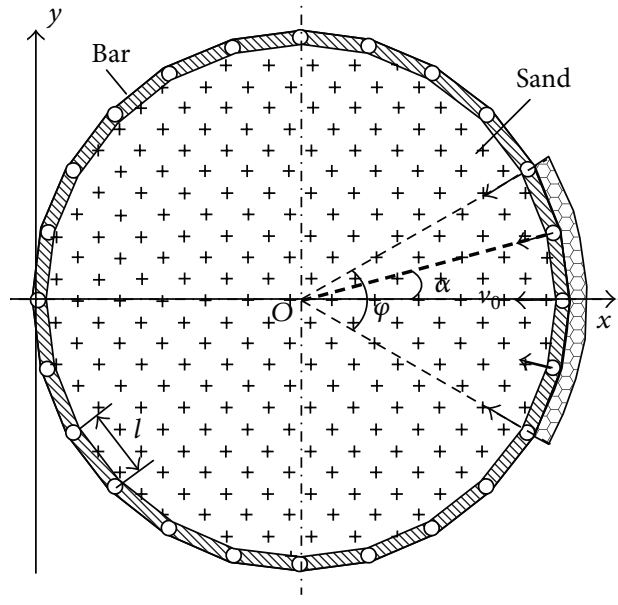

Figure 4: Analytical model.

The relationship between the bending moment and the deflection angle is expressed by the equation

$$
\theta= \begin{cases}\frac{M l}{E I} & M<M_{p} \\ \theta_{p} & M \geq M_{p}\end{cases}
$$

where $M$ is the bending moment; $I$ is the moment of inertia, which is equal to $t^{4} / 12 ; \theta$ is the deflection angle; $M_{p}$ is the plastic ultimate bending moment, which is equal to $t^{3} \sigma_{y} / 4$.

Moment of each square bar consists of two aspects: the moment generated by the spring force and the moment resulting from the bar bending. Moments of square bars are described by the equation

$$
\begin{aligned}
M_{h}(i)= & \left(\frac{l}{2}\right) \cdot F_{x}(i-1) \sin \alpha(i)-\left(\frac{l}{2}\right) \\
& \cdot F_{y}(i-1) \cos \alpha(i)+M(i), \\
M_{e}(i)= & \left(\frac{l}{2}\right) \cdot F_{x}(i) \sin \alpha(i)-\left(\frac{l}{2}\right) \\
& \cdot F_{y}(i) \cos \alpha(i)-M(i),
\end{aligned}
$$

where $M_{h}(i)$ and $M_{e}(i)$ are the moment generated by the spring force at the head and end of the current square bar, respectively. $M(i)$ is the moment generated by the relative bending between two adjacent square bars.

The internal medium is compressed because of the translation and rotation of square bars. During movement processes of all bars, interactions between square bars and internal medium are shown in Figure 7, where velocities of previous bar, current bar, and next bar are $v(i-1), v(i)$, and $v(i+1)$, respectively. The resistance forces suffered by the internal medium are $f(i-1), f(i)$, and $f(i+1)$, respectively.

It is assumed that the processes of the internal medium compressed undergo two stages. The first stage is that voids of internal medium are compacted, and the resistance force of each square bar is equal to $\rho_{e} t^{2}(\vec{v} \cdot \vec{n} l) \vec{v}$, where $\rho_{e}$ is the internal medium density. The second stage is that medium compacted suffers a shock compression, and the relationship between shock pressure and volume is assumed to be linear:

$$
p=\frac{\eta\left(d V-j_{0} \cdot V_{0}\right)}{\left(1-j_{0}\right) \cdot V_{0}}
$$

where $p$ is the shock pressure; $\eta$ is a scale factor; $V_{0}$ and $d V$ are the initial volume and the compression volume of internal medium, respectively; $j_{0}$ is the compression ratio of medium.

The resistance forces of bar generated by internal medium during the two stages are expressed by the equation

$$
\vec{f}= \begin{cases}\rho_{e} t^{2}(\vec{v} \cdot \vec{n} l) \vec{v} & j<j_{0} \\ p t \cdot \vec{n} l & j \geq j_{0} .\end{cases}
$$

By calculating the spring forces between adjacent square bars and the resistance forces generated by the internal medium, translational accelerations of all square bars are obtained at corresponding time. By calculating the bending moments of all square bars, the rotational accelerations are obtained at corresponding time. The translational and rotational accelerations of square bars are described by

$$
\begin{aligned}
\vec{a}(i) & =\frac{\vec{f}(i)+\vec{F}(i-1)+\vec{F}(i)}{m}, \\
\beta(i) & =\frac{M_{h}(i)+M_{e}(i)}{I},
\end{aligned}
$$

where $\alpha(i)$ and $\beta(i)$ are the translational and rotational accelerations of the current square bar, respectively.

Utilizing (8), the translational and rotational accelerations are calculated, which are the initial conditions at the next time step.

3.3. Initial Conditions. Initial translational acceleration, rotational acceleration, and velocities of all square bars are set to 0 , and the distributions of initial velocities are as follows:

$$
\vec{v}=\left\{\begin{array}{lc}
{\left[v_{0} \cos \alpha, v_{0} \sin \alpha\right]} & -\frac{\varphi}{2} \leq \alpha \leq \frac{\varphi}{2} \\
0 & \alpha<-\frac{\varphi}{2}, \quad \alpha>\frac{\varphi}{2},
\end{array}\right.
$$

where $\varphi$ is the half angle covered by the lateral explosive charge, $\alpha$ is the angle between the current square bar and $x$ axis, and $v_{0}$ is the initial velocity of cylindrical shell close to the lateral explosive charge.

According to the Gurney equations on contact explosion, the ring velocity can be obtained by using the equation [17]

$$
v_{0}=\sqrt{2 E} \sqrt{\frac{3}{1+5 M_{f} / C_{e}+4\left(M_{f} / C_{e}\right)^{2}}},
$$

where $v_{0}$ is the ring velocity $(\mathrm{m} / \mathrm{s}) ; \sqrt{2 E}$ is the Gurney energy unit mass $(\mathrm{m} / \mathrm{s}) ; C_{e}$ is the mass of lateral explosive charge (kg); $M_{f}$ is the mass of the cylindrical shell close to the lateral charge $(\mathrm{kg})$. The Gurney equation is a classical method to solve the metal velocity in recent decades, the error between the experimental and calculated results is about $5 \%$, and its accuracy is acceptable for our work. 

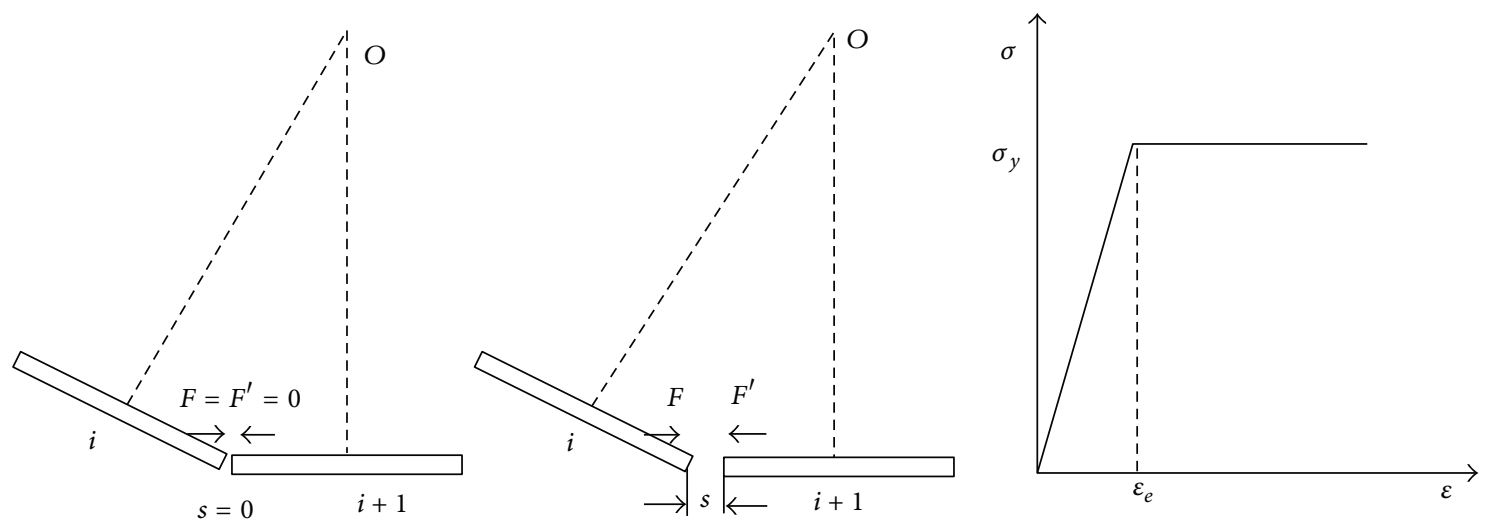

FIGURE 5: Spring force between two adjacent square bars.
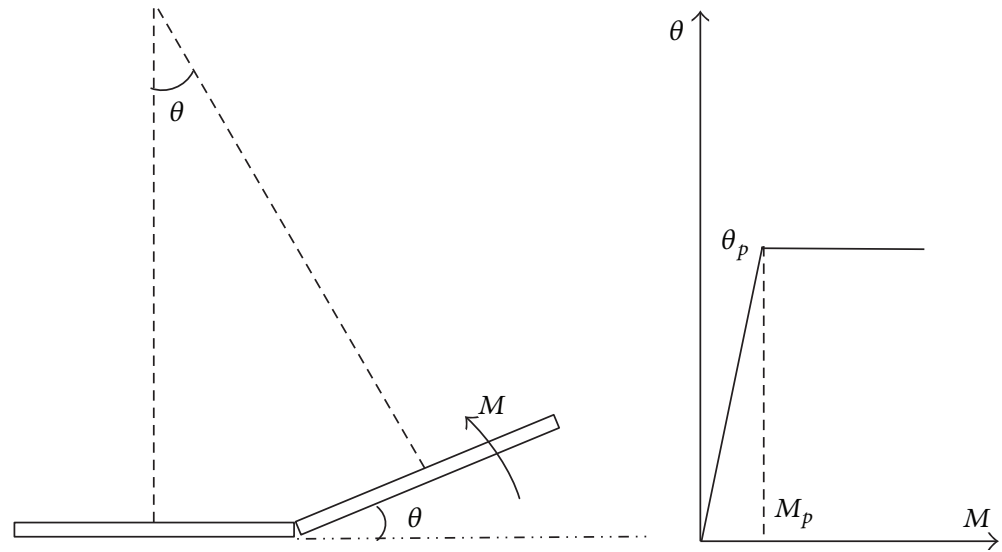

FIGURE 6: Bending moment and deflection angle between bars.

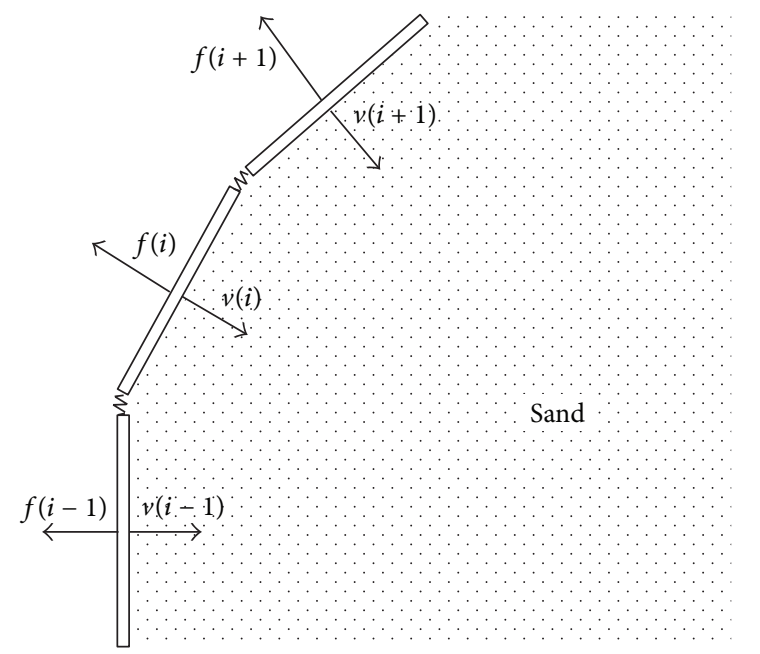

FIGURE 7: Interactions between square bars and internal medium.

\section{Calculated Results}

According to specific structure parameters and the type of the explosive charge, the velocity of the ring can be obtained with (8). It is assumed that the ring, made of 1020 Steel, has a thickness of $2 \mathrm{~mm}$, and the explosive, made of DL103-80, has the thickness of $5 \mathrm{~mm}$. Based on detonation parameters of DL103-80, substituting these parameters into the Gurney equation, the velocity is approximately $200 \mathrm{~m} / \mathrm{s}$. If the explosive DL103-80 is replaced with RDX or HMX, the velocity is greater, and the different initial velocity distribution can be obtained by varying the explosive charge material and the explosive mass.

Based on the analytical approach, calculated results are reported and discussed. Deformation processes of the cylindrical shell consist of two stages: stage I, velocities of the cylindrical shell obtained by the lateral contact explosion loading, and stage II, interactions between the cylindrical shell and internal medium. Figure 8 illustrates distribution of positions and velocities of the cylindrical shell at five different moments under the covered width $45^{\circ}$ and the initial velocity of the cylindrical shell $200 \mathrm{~m} / \mathrm{s}$, respectively. Figures 9 and 10 show distributions of positions and velocities of the cylindrical shell at five classical times under the covered widths $90^{\circ}$ and $135^{\circ}$, respectively. The ultimate translational velocities of the cylindrical shell are $6.1 \mathrm{~m} / \mathrm{s}, 7.3 \mathrm{~m} / \mathrm{s}$, and $10.9 \mathrm{~m} / \mathrm{s}$ with the covered widths $45^{\circ}, 90^{\circ}$, and $135^{\circ}$, respectively.

From the results of deformation shapes, a good agreement has been obtained between calculated and experimental results, and thus the analytical approach can be considered as 

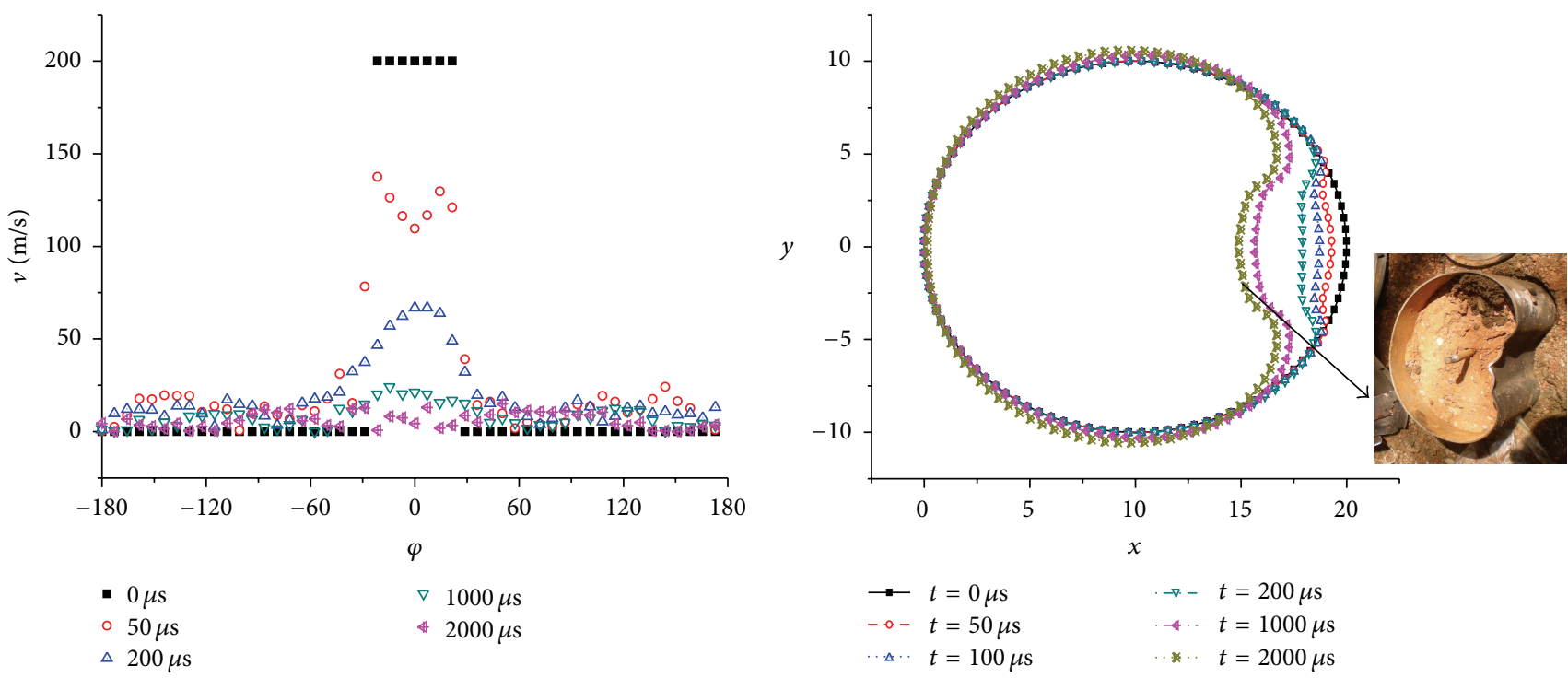

FIGURE 8: Distributions of positions and velocities of the cylindrical shell at different moments $\left(\varphi=45^{\circ}\right)$.
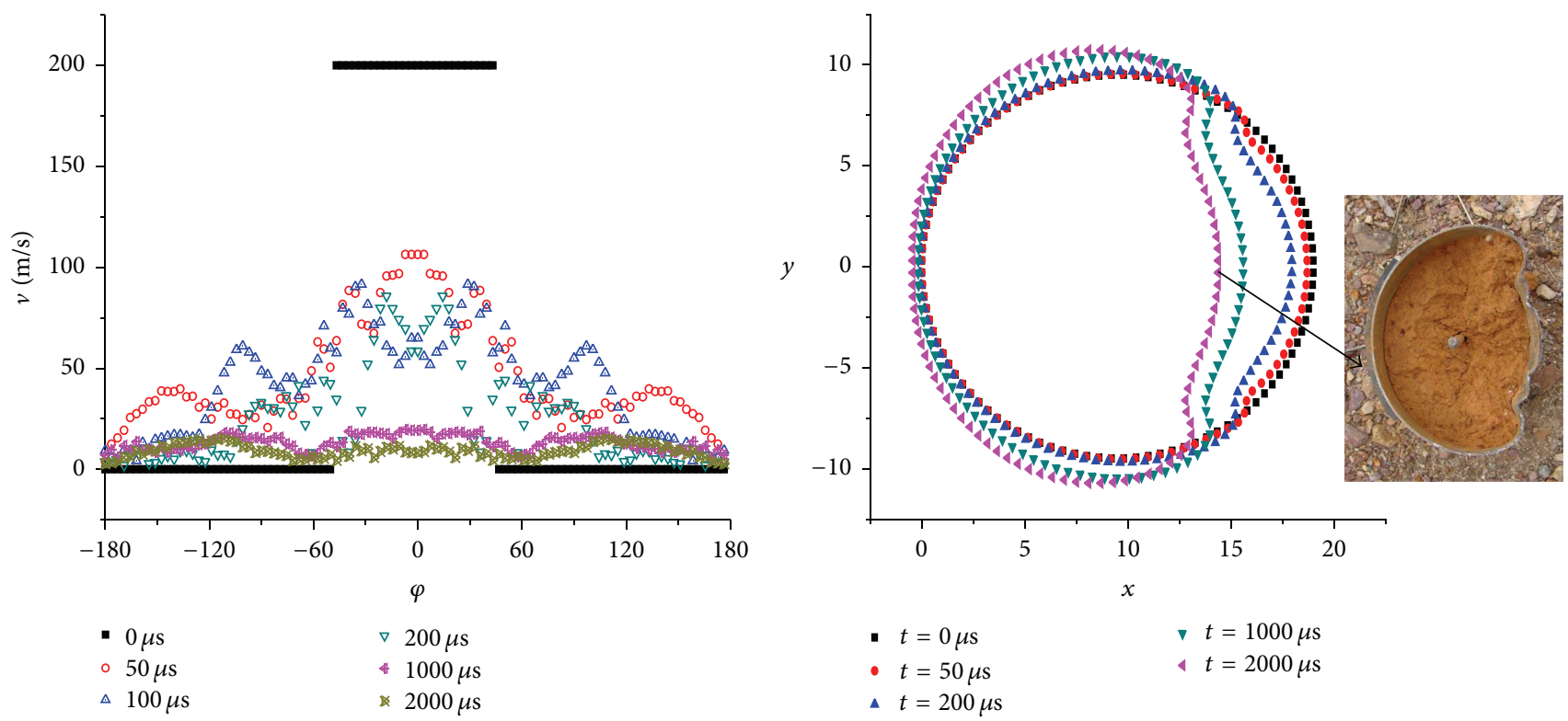

FIGURE 9: Distributions of positions and velocities of the cylindrical shell at different moments $\left(\varphi=90^{\circ}\right)$.

a valuable tool in understanding the deformation mechanism and predicting the deformation shape of the cylindrical shell under lateral contact explosion loading.

\section{Parametric Studies}

Deformation shapes of the ring have a significant relationship with the covered width of lateral explosive, explosive materials, and initial velocities distribution. In order to better understand the deformation mechanism, parametric studies are carried out for the deformation shapes and corresponding results were discussed.
5.1. Effect of Covered Width of Lateral Explosive. From the calculated and experimental results, it is obvious that the covered width of the lateral explosive charge is a key factor to the deformation shapes. Deformation shapes of various covered widths of the lateral charge are shown in Figure 11, where initial velocity of the ring equals $200 \mathrm{~m} / \mathrm{s}$. Various deformation shapes can be achieved by changing the width of lateral charge.

5.2. Effect of Lateral Explosive Materials. In order to investigate the effect of lateral charge, a series of calculated results are obtained by adjusting various initial velocities 

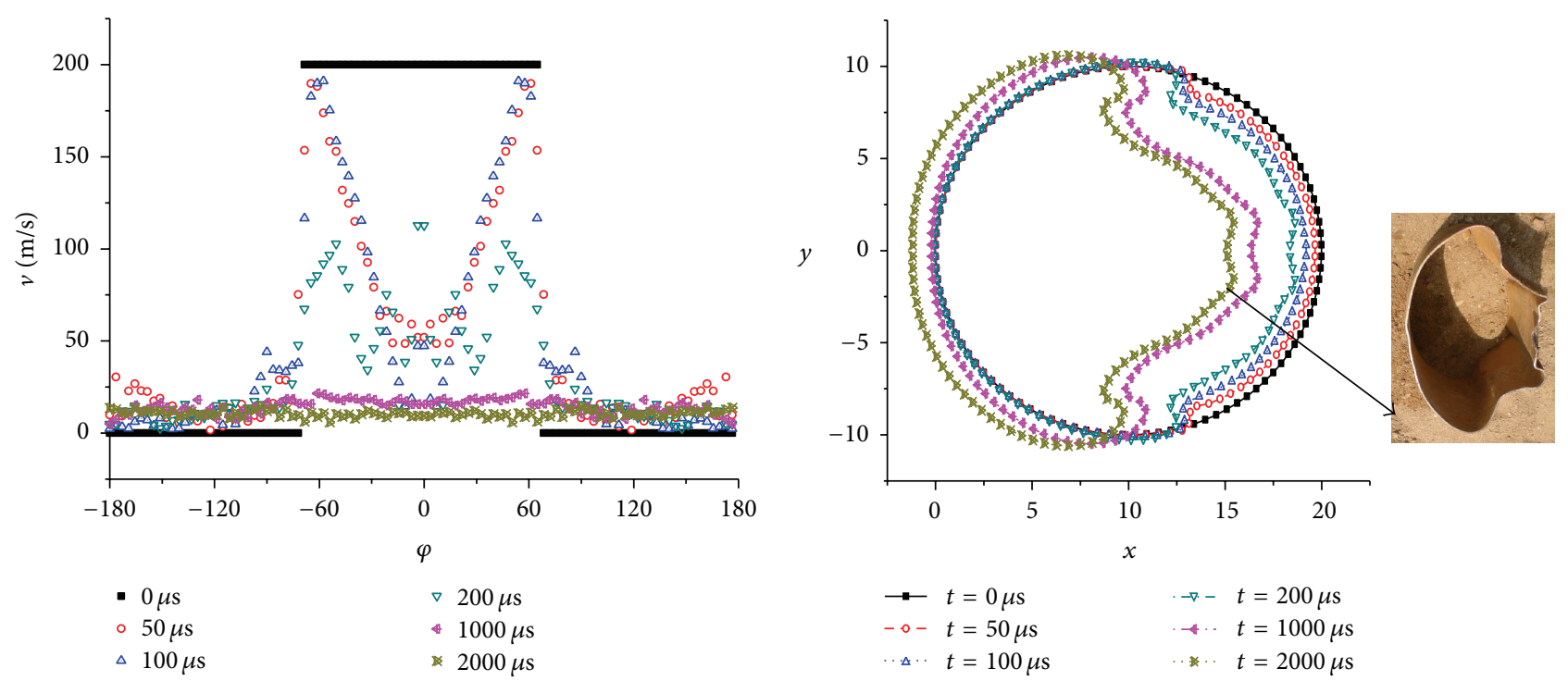

FIGURE 10: Distributions of positions and velocities of the cylindrical shell at different moments $\left(\varphi=135^{\circ}\right)$.

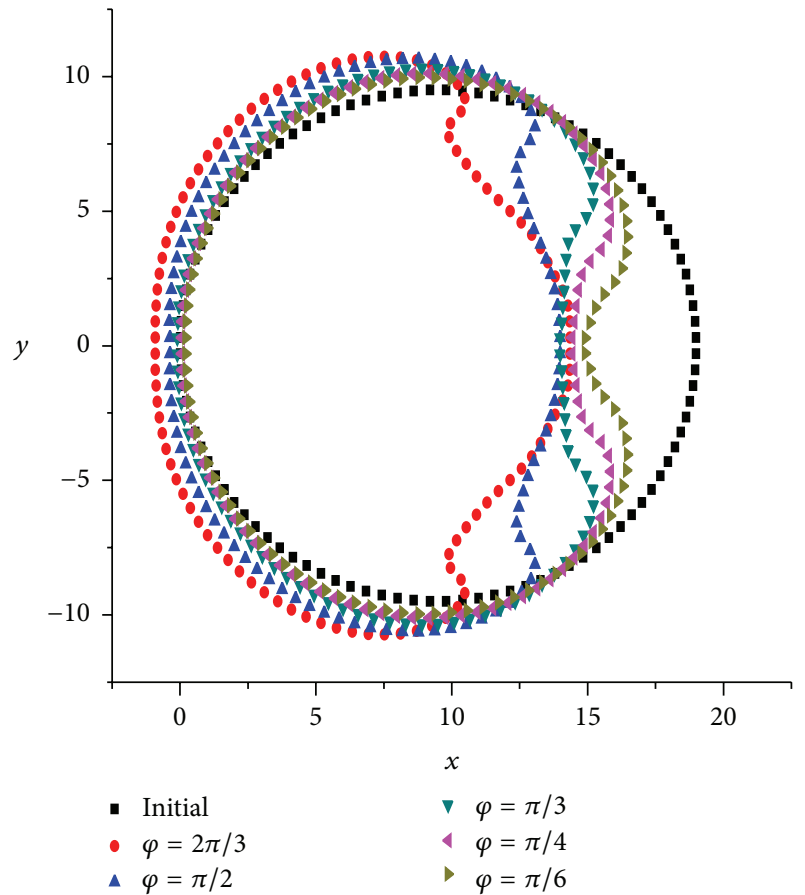

FIGURE 11: Deformation shapes of the cylindrical shell at various widths of lateral explosive charge $\left(v_{0}=200 \mathrm{~m} / \mathrm{s}\right)$.

of the cylindrical shell, because higher initial velocities of cylindrical shell represent greater power of charge.

Figure 12 shows the deformation shapes of the cylindrical shell at various initial velocities with the covered width $60^{\circ}$, in which velocities are $50 \mathrm{~m} / \mathrm{s}, 100 \mathrm{~m} / \mathrm{s}, 150 \mathrm{~m} / \mathrm{s}, 200 \mathrm{~m} / \mathrm{s}$, $250 \mathrm{~m} / \mathrm{s}$, and $300 \mathrm{~m} / \mathrm{s}$, respectively. The compression capacity of the sand medium increases with initial velocities; while initial velocities reach a certain extent, there is little change in deformation shapes.

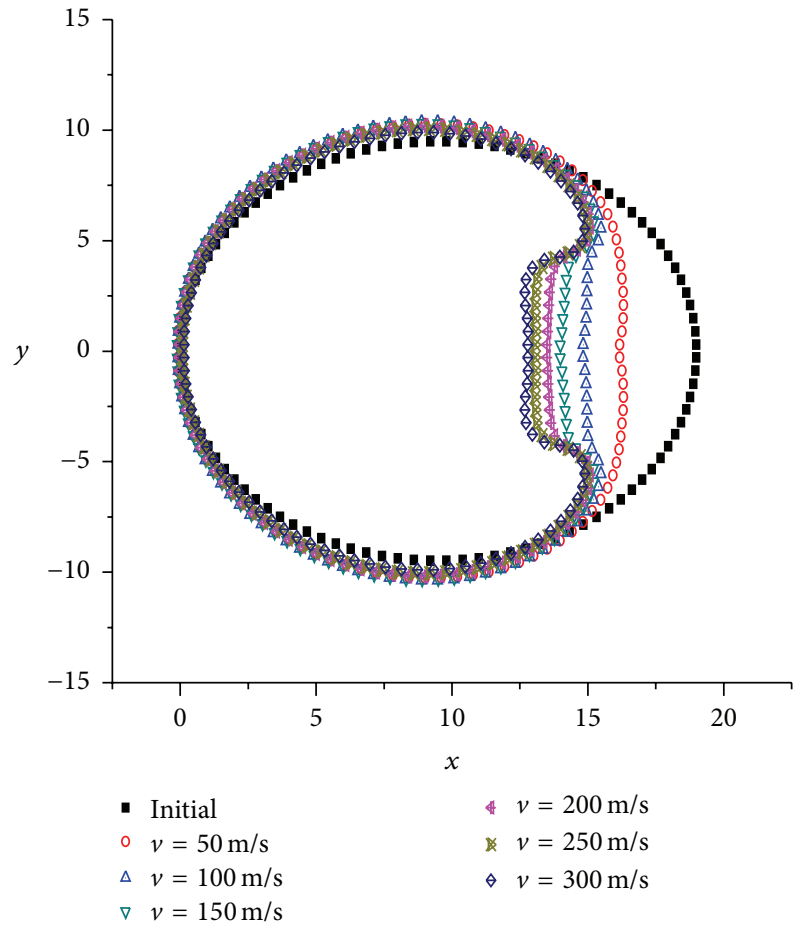

FIGURE 12: Deformation shapes of the cylindrical shell at various initial velocities $\left(\varphi=60^{\circ}\right)$.

5.3. Effect of Initial Velocities Distribution. In general, the lateral explosive charge has a uniform thickness in the circumferential direction within the central angle, and the ring close to the lateral explosive has the same velocity value. The velocity values varies with thickness of the circumferential explosive. The simplest assumption is that the thickness of the lateral explosive is a linear distribution from one side to 


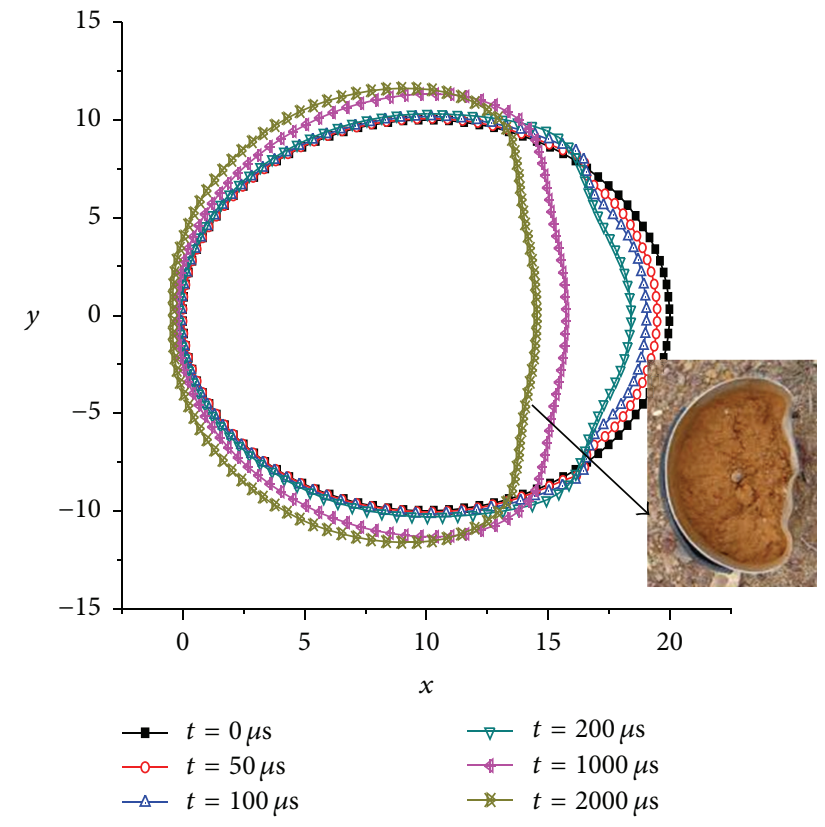

FIGURE 13: Distributions of positions and velocities of the cylindrical shell at different moments $\left(v(\alpha)=v_{0} \cos \alpha\right)$.

middle position, and the thickness of one side is a half of middle position.

Due to the explosive mass reduction, the initial velocities close to the lateral charge are assumed to have a cosine distribution. Figure 13 illustrates distribution of positions and velocities of the cylindrical shell at five classical times under the covered width $90^{\circ}$ and the initial velocities having a cosine distribution.

The plotted data in Figures 8-13 are from mathematical modeling, including deformation process, different covered width, and initial velocity, where there are several results compared with the experimental results. For example, in Figure 8, the deformation shape remains stationary at 2000 microsecond moment, which is in good agreement with the experimental results. From the calculated results of the velocity distributions of the ring, the velocity of each discrete bar is zero almost at 2000 microsecond moment, so it can represent the experimental result.

\section{Conclusions}

This paper presents brief results of an experimental investigation on the deformation process of the cylindrical shell with internal medium under lateral contact explosion, and the deformation shapes were obtained. Based on the experiments, a corresponding analytical approach has been undertaken using the rigid plastic hinge theory.

Given the structural parameters and explosive charge, deformation processes and shapes are reported using the analytical approach. A good agreement has been obtained between calculated and experimental results, and thus the analytical approach can be considered as a valuable tool in understanding the deformation mechanism and predicting the deformation shapes of the cylindrical shell with internal medium subjected to lateral contact explosion. Finally, a parametric study is carried out to analyze the effects of deformation shapes, depending on the covered width of the lateral explosive, explosive materials, and distribution of initial velocities. Therefore, an optimal deformation shape can be achieved by adjusting the covered width of lateral and initial velocities distribution.

\section{Conflict of Interests}

The authors declare that there is no conflict of interests regarding the publication of this paper.

\section{Acknowledgment}

The authors wish to acknowledge, with thanks, the financial support from the China National Natural Science Funding under Grants nos. 11202237 and 11132012.

\section{References}

[1] V. Hadavi, J. Z. Ashani, and A. Mozaffari, "Theoretical calculation of the maximum radial deformation of a cylindrical shell under explosive forming by a new energy approach," Proceedings of the Institution of Mechanical Engineers Part C: Journal of Mechanical Engineering Science, vol. 226, no. 3, pp. 576-584, 2012

[2] R. G. Yakupov, "Plastic strains in a cylindrical shell under the effect of a spherical blast wave," Strength of Materials, vol. 14, no. 1, pp. 52-56, 1982.

[3] R. G. Yakupov, "Plastic deformations of a cylindrical shell under the action of a planar explosion wave," Journal of Applied Mechanics and Technical Physics, vol. 23, no. 4, pp. 579-584, 1982.

[4] P. R. Gefken, S. W. Kirkpatrick, and B. S. Holmes, "Response of impulsively loaded cylindrical shells," International Journal of Impact Engineering, vol. 7, no. 2, pp. 213-227, 1988.

[5] M. S. T. Fatt and T. Wierzbicki, "Damage of plastic cylinders under localized pressure loading," International Journal of Mechanical Sciences, vol. 33, no. 12, pp. 999-1016, 1991.

[6] T. Wierzbicki and M. S. H. Fatt, "Damage assessment of cylinders due to impact and explosive loading," International Journal of Impact Engineering, vol. 13, no. 2, pp. 215-241, 1993.

[7] J. Jiang and M. D. Olson, "Nonlinear dynamic analysis of blast loaded cylindrical shell structures," Computers \& Structures, vol. 41, no. 1, pp. 41-52, 1991.

[8] Q. M. Li and N. Jones, "Blast loading of a 'short' cylindrical shell with transverse shear effects," International Journal of Impact Engineering, vol. 16, no. 2, pp. 331-353, 1995.

[9] C. Pédron and A. Combescure, "Dynamic buckling of stiffened cylindrical shells of revolution under a transient lateral pressure shock wave," Thin-Walled Structures, vol. 23, no. 1-4, pp. 85-105, 1995.

[10] R. Rajendran and J. M. Lee, "A comparative damage study of airand water-backed plates subjected to non-contact underwater explosion," International Journal of Modern Physics B, vol. 22, no. 9-11, pp. 1311-1318, 2008. 
[11] C. F. Hung, B. J. Lin, J. J. Hwang-Fuu, and P. Y. Hsu, "Dynamic response of cylindrical shell structures subjected to underwater explosion," Ocean Engineering, vol. 36, no. 8, pp. 564-577, 2009.

[12] J. Li and J.-L. Rong, "Experimental and numerical investigation of the dynamic response of structures subjected to underwater explosion," European Journal of Mechanics B/Fluids, vol. 32, no. 1, pp. 59-69, 2012.

[13] L.-J. Li, W.-K. Jiang, and Y.-H. Ai, "Experimental study on deformation and shock damage of cylindrical shell structures subjected to underwater explosion," Proceedings of the Institution of Mechanical Engineers Part C: Journal of Mechanical Engineering Science, vol. 224, no. 11, pp. 2505-2514, 2010.

[14] G. Y. Hu, G. Xia, and J. Li, “The transient responses of twolayered cylindrical shells attacked by underwater explosive shock waves," Composite Structures, vol. 92, no. 7, pp. 1551-1560, 2010.

[15] X.-L. Yao, J. Guo, L.-H. Feng, and A.-M. Zhang, "Comparability research on impulsive response of double stiffened cylindrical shells subjected to underwater explosion," International Journal of Impact Engineering, vol. 36, no. 5, pp. 754-762, 2009.

[16] H. H. Jama, G. N. Nurick, M. R. Bambach, R. H. Grzebieta, and X. L. Zhao, "Steel square hollow sections subjected to transverse blast loads," Thin-Walled Structures, vol. 53, pp. 109-122, 2012.

[17] M. A. Meyer, Dynamic Behavior of Materials, John Wiley \& Son, New York, NY, USA, 1994. 


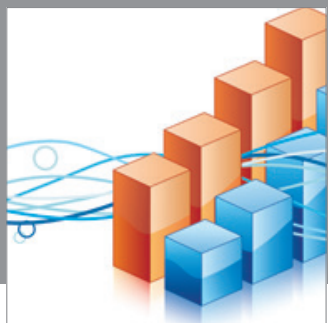

Advances in

Operations Research

mansans

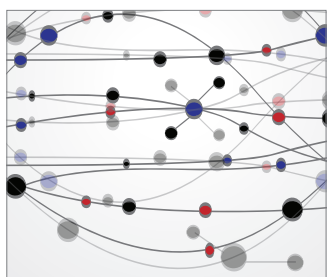

The Scientific World Journal
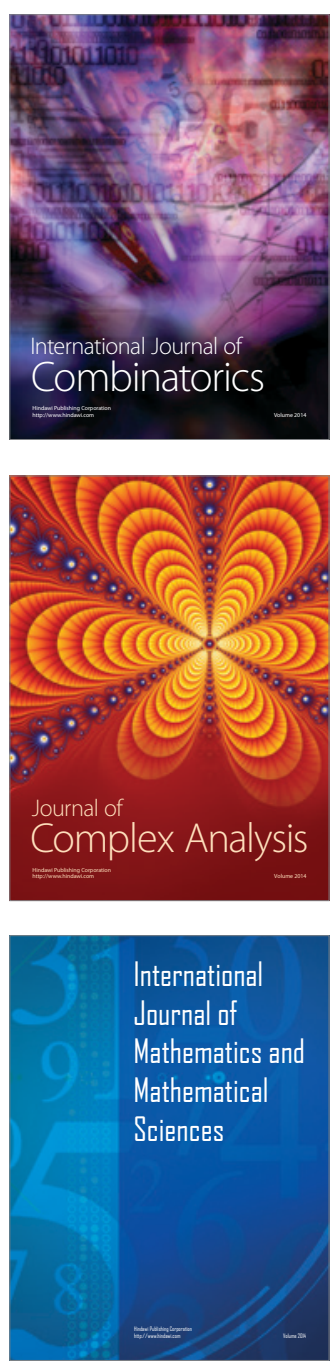
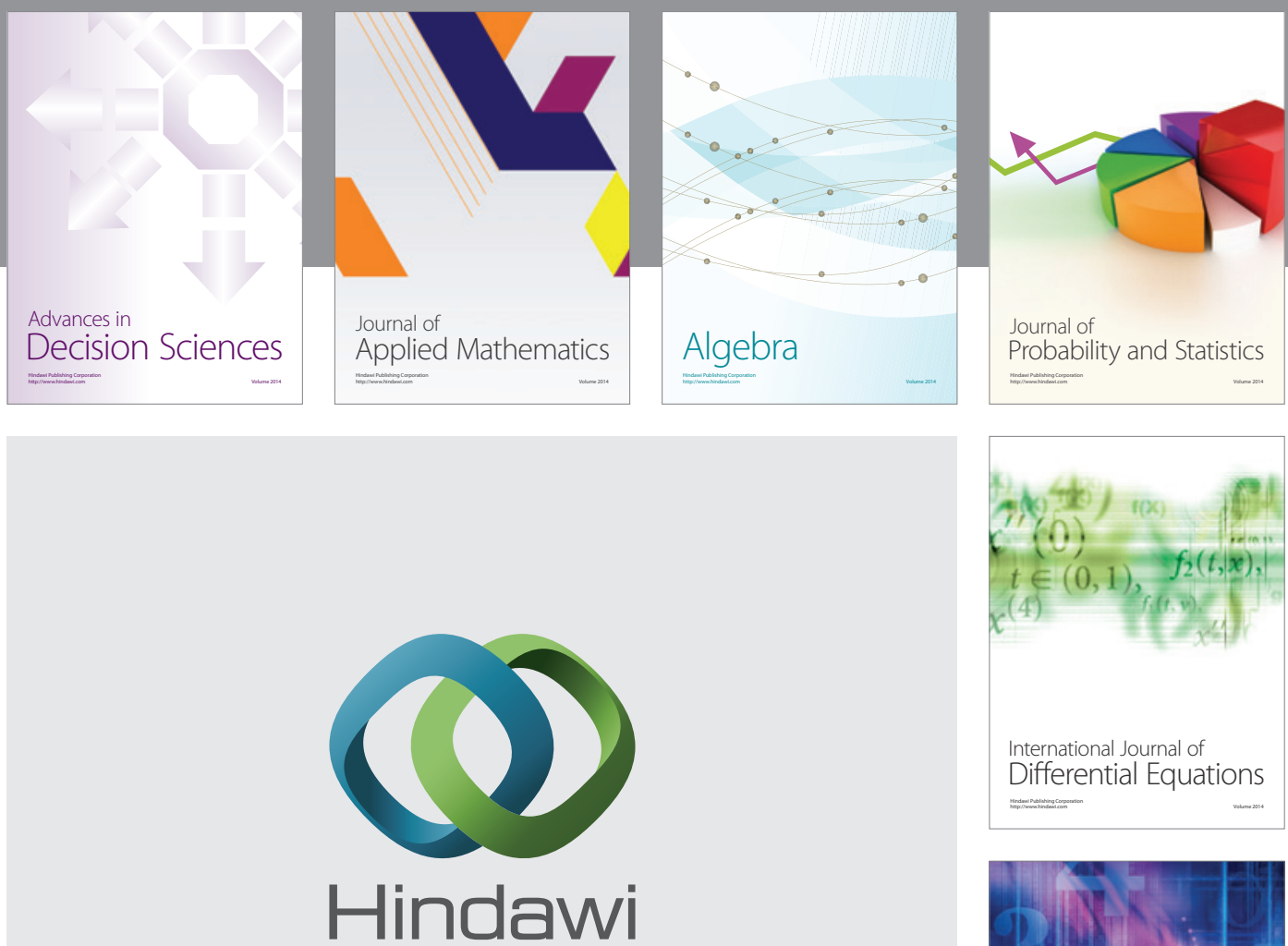

Submit your manuscripts at http://www.hindawi.com
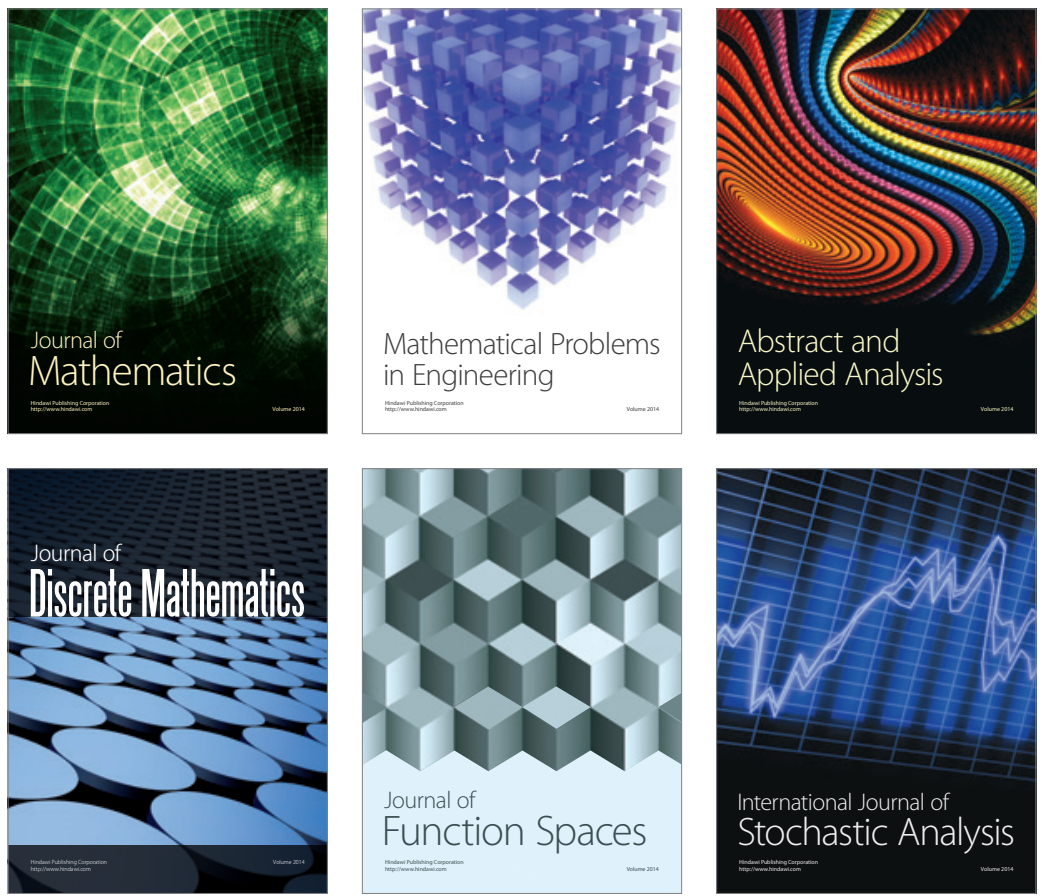

Journal of

Function Spaces

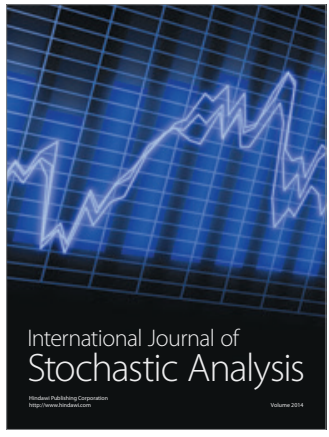

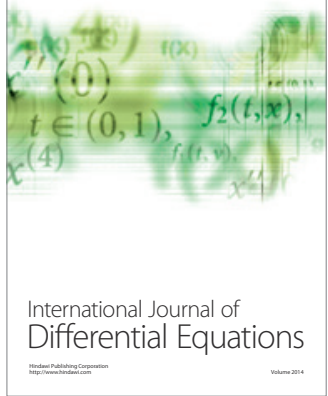
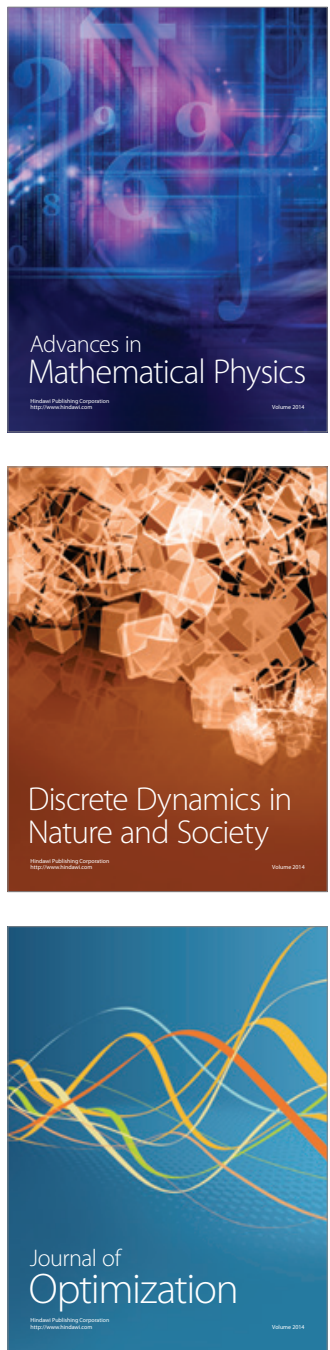